\title{
Efficiency of Rate-Maximization Game Under Bounded Channel Uncertainty
}

\author{
Amod J.G. Anandkumar ${ }^{\dagger}$, Animashree Anandkumar ${ }^{\ddagger}$, Sangarapillai Lambotharan ${ }^{\dagger}$ and Jonathon Chambers ${ }^{\dagger}$. \\ ${ }^{\dagger}$ Advanced Signal Processing Group, EE Dept., Loughborough University, Loughborough, UK LE11 3 TU. \\ ${ }^{\ddagger}$ Center for Pervasive Communications and Computing, EECS Dept., University of California Irvine \\ Email: ${ }^{\dagger}\left\{\right.$ A.J.G.Anandkumar, S.Lambotharan, J.A.Chambers\}@lboro.ac.uk, ${ }^{\ddagger}$ a.anandkumar@uci.edu
}

\begin{abstract}
The problem of competitive rate-maximization is an important signal-processing problem for power-constrained multi-user systems. It involves solving the power control problem for mutually interfering users operating across multiple frequencies. We introduced robust rate-maximization game for systems with bounded channel uncertainty. In this paper, we analyse the effect of uncertainty on the global efficiency of the robust rate-maximization game. For a two-user scenario with large number of frequencies, we show that the robustoptimization equilibrium tends to move towards FDMA solution as the uncertainty bound increases and thus increases the sumrate for interference-constrained systems where FDMA is Paretooptimal. These results are verified through simulations.
\end{abstract}

\section{INTRODUCTION}

The use of game theory as a tool for the analysis and study of communications problems has grown over the last decade [1]. Game theory provides useful insight into the design and analysis of multi-agent systems where the actions of one user affects the payoffs of other users. Typically, multi-agent systems involve multi-objective optimization problems which are analytically complex and difficult to solve, especially using distributed algorithms. The lack of equilibrium in such systems will mean that they are inherently unstable. Game theory provides a strong framework of well-defined equilibrium conditions under various conditions. The Nash equilibrium (NE) is a particularly useful result for systems where the users compete with each other [2].

The sequential iterative waterfilling algorithm for maximizing information rates in digital subscriber line systems [3] was one of the earliest works which used a game-theoretic approach to design a decentralized algorithm for multi-user dynamic power control. At any Nash equilibrium of this ratemaximization game, given that the power allocations of other users is constant, no user can further increase the achieved information rate unilaterally. However, this work and others extending this work such as [4]-[7] all assume perfect channel state information. This is a very strong requirement and cannot be met by practical systems.

The traditional game-theoretic solution for systems with imperfect information is the Bayesian game model [2] which uses a probabilistic approach to model the uncertainty in the system. However, a Bayesian approach is often intractable and the results strongly depend on the nature of the probability distribution functions. Techniques to define the bounded uncertainty sets in specific distributed optimization problems in communication networks so that they can be solved distributively by robust-optimization solutions are presented in [8]. A distribution-free robust game model for incompleteinformation finite games where the players use a robust optimization approach to counter bounded payoff uncertainty is presented in [9]. This robust game model also introduced a distribution-free equilibrium concept called the robustoptimization equilibrium. However, the results in [9] for the robust game model are limited to finite games, which is not applicable here.

A numerical solution to a robust optimization approach for the rate-maximization game with uncertainty is briefly considered in [10]. A robust iterative waterfilling algorithm based on a probabilistically constrained optimization approach for a system where the noise-plus-interference levels are quantized is presented in [11]. A robust waterfilling algorithm for a cognitive radio scenario with uncertainty in the primary channel is presented in [12].

We presented a distribution-free robust game formulation for the rate-maximization game where non-cooperative users formulate best responses to worst-case interference to counter channel uncertainty [13]. In this work we analyse the global efficiency of the robust rate-maximization game and present conditions when increasing uncertainty will result in increasing sum-rate.

This paper is organized as follows: Section II describes the system model and necessary preliminaries. Section III is a review of the robust rate-maximization game under bounded channel uncertainty [13]. Section IV presents the analysis of efficiency of the equilibrium for the two-user case under a large number of frequencies. Section V presents the simulation results and finally Section VI draws the conclusions from this work.

Notations used: The expectation operator is denoted by $\mathrm{E}\{\cdot\}$. The quantity $[\mathbf{A}]_{i j}$ refers to the $(i, j)$-th element of $\mathbf{A}$. $\mathbb{R}_{+}^{m \times n}$ is the set of $m \times n$ matrices with real non-negative elements. The spectral radius (largest eigenvalue) of matrix A is denoted by $\rho(\mathbf{A})$ [14]. The operation $[x]_{a}^{b}$ is defined as $[x]_{a}^{b}=a$ if $x \leq a ; x$ if $a<x<b ; b$ if $x \geq b$.

\section{SySTEM MODEL}

The system model is described in detail in [?]. In brief, we have a frequency-selective Gaussian interference channel with $N$ frequencies, composed of $Q$ SISO links, where $H_{r q}(k)$ 
is the normalized frequency response of the $\mathrm{k}$-th frequency bin of the channel between source $r$ and destination $q$ and $\sigma_{q}^{2}(k)$ is the normalized noise variance at receiver $q$ in the kth frequency bin. The normalized power allocated to the $k$-th frequency bin by user $q$ is $p_{q}(k)$. The power allocation vector of each user $q$ has two constraints: (a) Maximum total transmit power for each user, $\frac{1}{N} \sum_{k=1}^{N} p_{q}(k) \leq 1$; (b) Spectral mask constraints, $p_{q}(k) \leq p_{q}^{\max }(k)$. The power allocation vectors are public information, i.e. known to all users.

The channel between itself and all the transmitters is estimated by each receiver, which is private information known only to itself. The power allocation vectors of all users is public information, i.e. known to all users. The optimal power allocation across the frequency bins for its own link is computed by each receiver and sent back to the corresponding transmitter in a low bit-rate error-free feedback channel. Note that this leads to sharing of more information compared to other works in literature such as [6]. The channel state information estimated by each receiver is assumed to have a bounded uncertainty of unknown distribution, which is approximated to be an ellipsoid uncertainty set [15]. We consider that at each frequency, the uncertainty in the channel state information of each user is deterministically modelled under an ellipsoid approximation as

$$
\mathcal{F}_{q}=\left\{F_{r q}(k)+\Delta F_{r q, k}: \sum_{r \neq q}\left|\Delta F_{r q, k}\right|^{2} \leq \epsilon_{q}^{2}\right\},
$$

for $k=1, \ldots, N$, where $\epsilon_{q} \geq 0 \forall q \in \Omega$ is the uncertainty bound and

$$
F_{r q}(k) \triangleq \frac{\left|H_{r q}(k)\right|^{2}}{\left|H_{q q}(k)\right|^{2}},
$$

with $F_{r q}(k)$ being the nominal value. We can consider uncertainty in $F_{r q}$ instead of $H_{r q}(k)$ since bounded uncertainties in $F_{r q}(k)$ and $H_{r q}(k)$ are equivalent, but with different bounds.

The nominal information rate (i.e. assuming zero uncertainty) of user $q$ can be written as [16]

$$
R_{q}=\sum_{k=1}^{N} \log \left(1+\frac{p_{q}(k)}{\sigma_{q}^{2}(k)+\sum_{r \neq q} F_{r q}(k) p_{r}(k)}\right),
$$

"Inefficiency" of the equilibria of a game are often characterized using the price of anarchy and the price of stability of the equilibrium. The price of anarchy is defined as the ratio between the objective function value at the socially optimal solution and the worst objective function value at any equilibrium of the game [17]. The price of stability is defined as the ratio between the objective function value at the socially optimal solution and the best objective function value at any equilibrium of the game [17]. We consider the sum-rate of the system to be our social objective function. The sum-rate of the system is given by

$$
S=\sum_{q=1}^{Q} R_{q}
$$

In our case, the price of stability and anarchy are the same due to the existence of a unique equilibrium as shown in
Theorem 1. Thus, the price of anarchy, PoA, is the ratio of the sum-rate of the system at the social optimal solution, $S^{*}$, and the sum-rate of the system at the robust-optimization equilibrium, $S^{\text {rob }}$, i.e.,

$$
\mathrm{PoA}=\frac{S^{*}}{S^{\mathrm{rob}}}
$$

Note that a more efficient equilibrium has a lower price of anarchy.

\section{OVERVIEW OF ROBUST RATE-MAXIMIZATION GAME}

In this section, we present a brief overview of the robust rate-maximization game that was introduced in [13]. In the robust rate-maximization game, each user formulates the best response while considering the worst-case multi-user interference that could be observed, given the strategies of the other users. The robust rate-maximization game can be mathematically defined as, $\forall q \in \Omega$,

$$
\begin{aligned}
& \max _{\mathbf{p}_{q}} \sum_{k=1}^{N} \log \left(1+\frac{p_{q}(k)}{\sigma_{q}^{2}(k)+\sum_{r \neq q} F_{r q}(k) p_{r}(k)+\epsilon_{q} \sqrt{\sum_{r \neq q} p_{r}^{2}(k)}}\right) \\
& \text { s.t. } \quad \mathbf{p}_{q} \in \mathcal{P}_{q} .
\end{aligned}
$$

where $\mathcal{P}_{q}$ is the set of admissible strategies of user $q$, which is defined as

$$
\begin{aligned}
\mathcal{P}_{q} \triangleq\left\{\mathbf{p}_{q} \in \mathbb{R}^{N}: \frac{1}{N} \sum_{k=1}^{N} p_{q}(k)=1,\right. & \\
& \left.0 \leq p_{q}(k) \leq p_{q}^{\max }(k), k=1, \ldots, N\right\} .
\end{aligned}
$$

The solution to this game is the robust-optimization equilibrium (RE). At any robust-optimization equilibrium of this game, the optimum action profile of the players $\left\{\mathbf{p}_{q}^{\star}\right\}_{q \in \Omega}$ must satisfy the following set of simultaneous waterfilling equations: $\forall q \in \Omega$,

$$
\mathbf{p}_{q}^{\star}=\mathrm{RWF}_{\mathrm{q}}\left(\mathbf{p}_{-q}^{\star}\right) .
$$

where $\left[\operatorname{RWF}_{\mathrm{q}}\left(\mathbf{p}_{-q}\right)\right]_{k} \triangleq$

$$
\left[\mu_{q}-\sigma_{q}^{2}(k)-\sum_{r \neq q} F_{r q}(k) p_{r}(k)-\epsilon_{q} \sqrt{\sum_{r \neq q} p_{r}^{2}(k)}\right]_{0}^{p_{q}^{\max }(k)}
$$

The robust waterfilling operation for each user is a distributed worst-case optimization under bounded channel uncertainty. We can see that an additional term has appeared in (9) for $\epsilon_{q}>0$ when compared with the original waterfilling operation in [6] under perfect CSI (i.e. $\epsilon_{q}=0 \forall q$ ).

This additional term acts as a penalty against allocating power to frequencies that have a large product of the uncertainty bound and the norm of the powers of the other players currently transmitting in those frequencies. This is caused by the users assuming the worst-case interference from other users and are thus being conservative about allocating power 
to such channels where there is a strong presence of other users.

Let $\mathcal{N}=\{1, \ldots, N\}$ be the set of frequency bins. Let $\mathcal{D}_{q}^{\circ}$ denote the set of frequency bins that user $q$ would never use as the best response to any set of strategies adopted by the other users,

$$
\begin{aligned}
\mathcal{D}_{q}^{\circ} \triangleq\{k \in & \{1, \ldots, N\}: \\
& {\left.\left[\operatorname{RWF}_{\mathrm{q}}\left(\mathbf{p}_{-q}\right)\right]_{k}=0 \quad \forall \mathbf{p}_{-q} \in \mathcal{P}_{-q}\right\} }
\end{aligned}
$$

where $\mathcal{P}_{-q} \triangleq \mathcal{P}_{1} \times \cdots \times \mathcal{P}_{q-1} \times \mathcal{P}_{q+1} \times \cdots \times \mathcal{P}_{Q}$. The nonnegative matrices $\mathbf{E}$ and $\mathbf{S}^{\max } \in \mathbb{R}_{+}^{Q \times Q}$ are defined as

$$
[\mathbf{E}]_{q r} \triangleq\left\{\begin{array}{lr}
\epsilon_{q}, & \text { if } r \neq q, \\
0, & \text { otherwise }
\end{array}\right.
$$

and

$$
\left[\mathbf{S}^{\text {max }}\right]_{q r} \triangleq\left\{\begin{array}{lr}
\max _{k \in \mathcal{D}_{q} \cap \mathcal{D}_{r}} F_{r q}(k), & \text { if } r \neq q, \\
0, & \text { otherwise }
\end{array}\right.
$$

where $\mathcal{D}_{q}$ is any subset of $\{1, \ldots, N\}$ such that $\mathcal{N}-\mathcal{D}_{q}^{\circ} \subseteq$ $\mathcal{D}_{q} \subseteq\{1, \ldots, N\}$.

The sufficient condition for existence and uniqueness of the $\mathrm{RE}$ of game $\mathscr{G}^{\mathrm{rob}}$ is given by the following theorem [13]:

Theorem 1: Game $\mathscr{G}^{\mathrm{rob}}$ has at least one equilibrium for any set of channel matrices and transmit powers of the users. Furthermore, the equilibrium is unique if

$$
\rho\left(\mathbf{S}^{\max }\right)<1-\rho(\mathbf{E}),
$$

where $\mathbf{E}$ and $\mathbf{S}$ are as defined in (11) and (12) respectively.

This condition for the robust-optimization equilibrium reduces to condition $(\mathrm{C} 1)$ in [6] for the Nash equilibrium when there is no uncertainty in the system, i.e. when $\epsilon_{q}=0 \forall q \in \Omega$. Further, the set of channel coefficients for which the existence of a unique equilibrium is guaranteed shrinks as the uncertainty bound increases, since $\rho(E)>0$ when any $\epsilon_{q}>0$.

The robust asynchronous iterative waterfilling algorithm for computing the RE of game $\mathscr{G}^{\mathrm{rob}}$ in a distributed fashion is described in Algorithm 1. The convergence of Algorithm 1 is guaranteed under the following condition [13]:

Theorem 2: The asynchronous iterative waterfilling algorithm described in Algorithm 1 converges to the unique RE of game $\mathscr{G}^{\mathrm{rob}}$ as the number of iterations for which the algorithm is run, $T \rightarrow \infty$ for any set of feasible initial conditions if condition (13) is satisfied.

The global convergence of the distributed robust iterative waterfilling algorithm to the unique RE is guaranteed by Theorem 2 using condition (13) despite game $\mathscr{G}^{\mathrm{rob}}$ and the waterfilling operation $\operatorname{RWF}_{\mathrm{q}}(\cdot)$ being nonlinear. Further, the set of channel coefficients for which convergence of the algorithm is guaranteed reduces as the uncertainty bound increases.

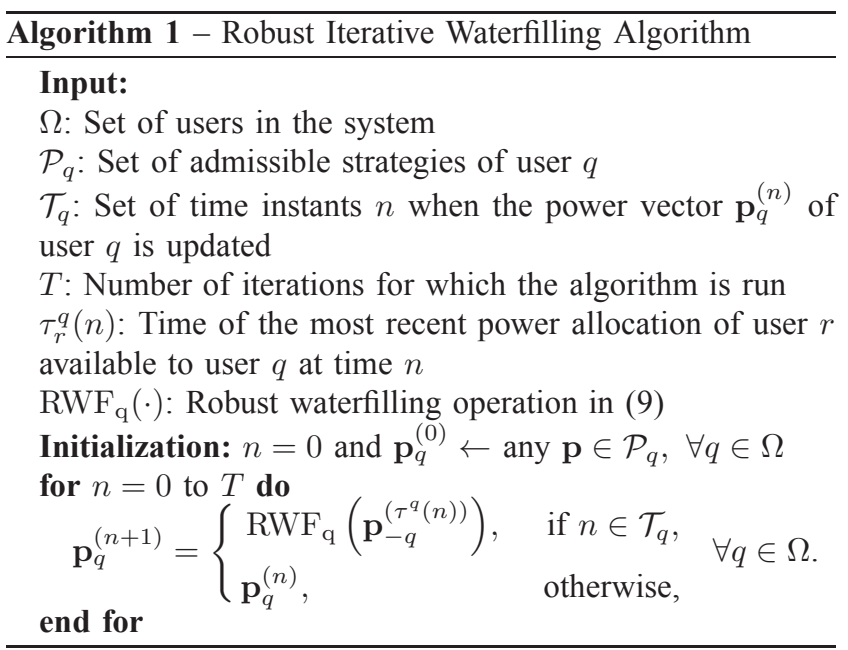

\section{EFFiciency at the Equilibrium - Two-User CASE}

In this section, we analyse the effect of varying uncertainty bounds on the efficiency of the system. When the system has only two users, the worst-case interference in each frequency reduces to $\left(F_{r q}(k)+\epsilon_{q}\right) p_{r}(k)$ with $q, r=1,2$ and $q \neq r$. This implies that the robust waterfilling operation for the two user case $(Q=2)$ is simply the standard waterfilling solution with the worst-case channel coefficients. We restrict our analysis to the two-user case where both the users have identical noise variances $\sigma_{q}^{2}(k)=\sigma^{2} \forall k, q$ across all frequencies, identical uncertainty bounds $\epsilon_{1}=\epsilon_{2}=\epsilon$ and equal total power constraints $\sum_{k=1}^{N} p_{1}(k)=\sum_{k=1}^{N} p_{2}(k)=1$.

The quantity $J(k)$, defined as

$$
J(k) \triangleq-p_{1}(k) p_{2}(k),
$$

is considered as a measure of the extent of partitioning of the frequency $k$. It is minimum $(J(k)=-1)$ when both the users allocate all their total power to the same frequency $k$ and is maximum $(J(k)=0)$ when at most one user is occupying the frequency $k$. Note that $J(k)=0 \forall k \in\{1, \ldots, N\}$ when the users adopt an frequency division multiple access (FDMA) scheme.

The following lemma describes the effect of the uncertainty bound on the extent of partitioning of the system:

Lemma 1: When the number of frequencies, $N \rightarrow \infty$, the extent of partitioning in every frequency is non-decreasing as the uncertainty bound of the system increases for any set of channel matrices, i.e.,

$$
\frac{\mathrm{d}}{\mathrm{d} \epsilon} J(k) \geq 0 \quad \forall k \in\{1, \ldots, N\} \text { when } N \rightarrow \infty
$$

with equality for frequencies where $J(k)=0$, where $J(k)$ is defined in (14).

Proof: See [18].

From this lemma, we can see that the robust-optimization equilibrium moves towards greater frequency-space partitioning as the uncertainty bound increases when there is a large number of frequencies in the system. Thus, the RE is moving 
closer to an FDMA solution under increased channel uncertainty. When the FDMA solution is globally optimal [19], this will lead to an improvement in the performance of the equilibrium. This is stated in the following theorem:

Theorem 3: As the number of frequencies, $N \rightarrow \infty$, the sum-rate (price of anarchy) at robust-optimization equilibrium of the system is non-decreasing (non-increasing) as the uncertainty bound increases if, $\forall k \in\{1, \ldots, N\}$,

$$
\left(F_{21}(k)-\epsilon\right)\left(F_{12}(k)-\epsilon\right)>\frac{1}{4}
$$

Proof: See [18].

In frequency-flat systems, all users have equal power allocation to all frequencies at the equilibrium when (13) is satisfied [7]. This is not dependent on the uncertainty in the CSI and thus leads to no change in the extent of partitioning. Thus, the sum-rate and the price of anarchy are not affected by varying uncertainty bounds. Also, the modified waterfilling operation in (9) can be applied as a pricing mechanism to improve the sum-rate in a system with no uncertainty considering $\epsilon$ is a design parameter.

\section{Simulation Results}

In this section, simulation results to analyze the impact of channel uncertainty on the robust-optimization equilibrium is presented. The simulation parameters are presented in Figure 1 . We study three important aspects, namely the percentage of convergence, the total information rate observed at the receivers and the additional number of frequencies with zero power allocations at the RE when compared to the NE against the percentage of uncertainty ${ }^{1} \delta$. We see that the simulation results follow theoretical results for asymptotic number of frequencies even when the number of frequencies is as small as $N=8$ frequencies.

In Figure 1a, the sum-rate at the Nash equilibrium is when the uncertainty is zero. The sum-rate at the Nash equilibrium (with perfect CSI) is less than the sum-rate at the robustoptimization equilibrium (with imperfect CSI). Further, this gap in performance increases as the uncertainty increases. This is because the users are more cautious about using frequencies with significant interference, thus reducing the total amount of interference in the system.

In Figure 1b, the total number of channels occupied per user at the robust-optimization equilibrium decreases as the uncertainty bound increases. The users are using smaller number of frequencies as a result of the better partitioning of the frequency-space among the users which reduces interference. This leads to the higher sum-rates as observed in Figure 1a. These simulation results also indicate that schemes similar to the RE can move closer to Pareto optimality for rate-maximization games (which is FDMA for interference constrained systems [19]).

In Figure 1c, we observe that as the uncertainty $\delta$ increases, the average number of iterations for convergence increases.

\footnotetext{
${ }^{1}(9)$ and (8) are in terms of absolute uncertainty $\epsilon$ while the simulations use relative uncertainty $\delta$. They are equivalent to one another.
}

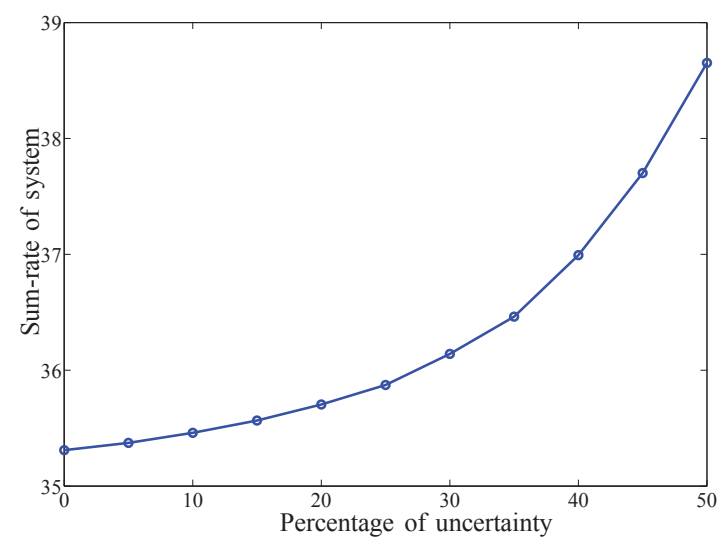

(a) Sum-rate of the system vs. uncertainty $\delta$.

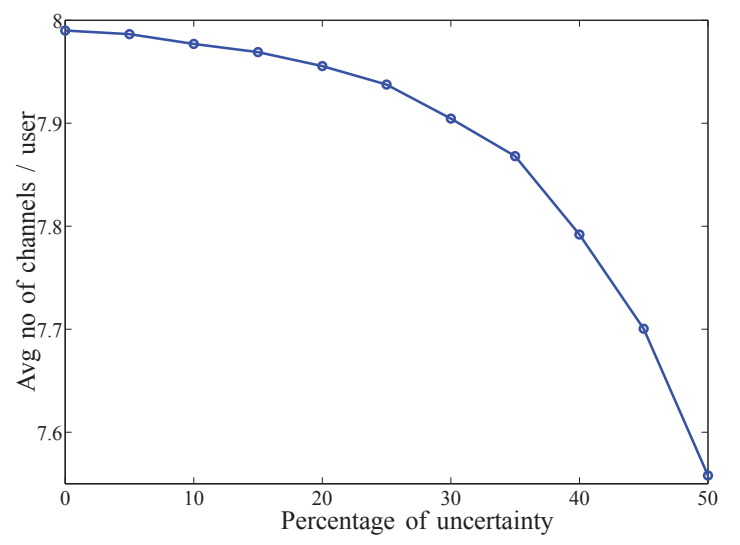

(b) Average number of channels occupied per user vs. uncertainty $\delta$.

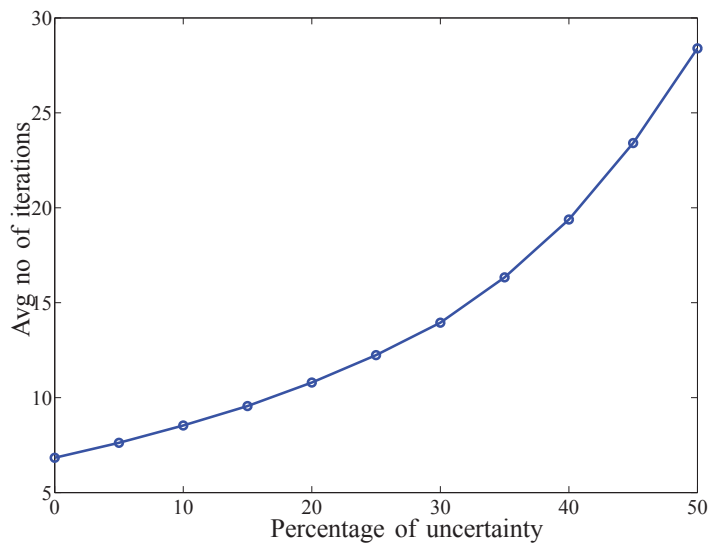

(c) Average number of iterations vs. uncertainty $\delta$.

Fig. 1: Simulation results for a system with $\sigma=0.1, Q=2$ users and $N=8$ frequencies over 1000 runs. Channel gains $H_{r q}(k) \sim \mathcal{C N}(0,1)$ for $r \neq q, H_{q q}(k) \sim \mathcal{C N}(0,4)$. Channel uncertainty model: nominal value $F_{r q}(k)=F_{r q}^{\text {true }}(k)(1+$ $\left.e_{r q}(k)\right)$ with $e_{r q}(k) \sim \mathcal{U}\left(-\frac{\delta}{2}, \frac{\delta}{2}\right), \delta<1$. The simulations are limited to channels which satisfy the sufficiency condition in (13). Note that the zero uncertainty corresponds to the Nash equilibrium 
This is as expected from [18, Lemma 1], as the modulus of the block-contraction increases as the uncertainty increases, which means that the step size of each iteration reduces as uncertainty increases, thus leading to slower convergence to the equilibrium.

\section{CONCLUSIONS}

In this paper, we have analyzed the efficiency of the previously introduced robust rate-maximization games for systems with bounded channel uncertainty. We analyze the effect of uncertainty on the sum-rate and price of anarchy of the system for the two-user scenario with large number of frequencies. We show that the robust-optimization equilibrium moves towards a frequency division multiple access (FDMA) solution as uncertainty bound increases and thus improves sum-rate for interference-constrained systems where FDMA is Pareto-optimal. This framework can be extended to MIMO rate-maximization games, cognitive radio and other non-cooperative games.

\section{REFERENCES}

[1] A. B. MacKenzie and L. A. DaSilva, "Game theory for wireless engineers," Synthesis Lectures on Communications, vol. 1, no. 1, pp. 186, 2006.

[2] M. J. Osborne and A. Rubinstein, A Course in Game Theory. MIT Press, 1999.

[3] W. Yu, G. Ginis, and J. Cioffi, "Distributed multiuser power control for digital subscriber lines," IEEE Journal on Selected Areas in Communications, vol. 20, pp. 1105-1115, Jun. 2002

[4] Z. Luo and J. Pang, "Analysis of iterative waterfilling algorithm for multiuser power control in digital subscriber lines," EURASIP Journal on Applied Signal Processing, vol. 6, pp. 1-10, 2006.

[5] K. Shum, K. Leung, and C. Sung, "Convergence of iterative waterfilling algorithm for Gaussian interference channels," IEEE Journal on Selected Areas in Communications, vol. 25, pp. 1091-1100, Aug. 2007.
[6] G. Scutari, D. Palomar, and S. Barbarossa, "Asynchronous iterative water-filling for Gaussian frequency-selective interference channels," IEEE Transactions on Information Theory, vol. 54, pp. 2868-2878, Jul. 2008.

[7] P. von Wrycza, M. R. B. Shankar, M. Bengtsson, and B. Ottersten, "Spectrum allocation for decentralized transmission strategies: properties of Nash equilibria," EURASIP Journal on Advances in Signal Processing, vol. 2009, pp. 1-11, 2009.

[8] K. Yang, Y. Wu, J. Huang, X. Wang, and S. Verdu, "Distributed robust optimization for communication networks," The 27th IEEE International Conference on Computer Communications (INFOCOM 2008), pp. 1157 1165, Apr. 2008.

[9] M. Aghassi and D. Bertsimas, "Robust game theory," Mathematical Programming, vol. 107, no. 1, pp. 231-273, 2006.

[10] P. Setoodeh and S. Haykin, "Robust transmit power control for cognitive radio," Proceedings of the IEEE, vol. 97, pp. 915 -939, May 2009.

[11] R. Gohary and T. Willink, "Robust IWFA for open-spectrum communications," IEEE Transactions on Signal Processing, vol. 57, pp. 4964 4970, Dec. 2009.

[12] J. Wang, G. Scutari, and D. Palomar, "Robust cognitive radio via game theory," 2010 IEEE International Symposium on Information Theory Proceedings (ISIT), pp. 2073 -2077, Jun. 2010.

[13] A.J.G. Anandkumar, A. Anandkumar, S. Lambotharan and J. Chambers, "Robust rate-maximization game under bounded channel uncertainty," 2010 IEEE International Conference on Acoustics, Speech, and Signal Processing (ICASSP), pp. 3158 -3161, Mar. 2010.

[14] R. A. Horn and C. R. Johnson, Matrix Analysis. Cambridge University Press, 1985.

[15] S. Boyd and L. Vandenberghe, Convex Optimization. Cambridge University Press, March 2004.

[16] T. M. Cover and J. A. Thomas, Elements of Information Theory. WileyInterscience, August 1991.

[17] N. Nisan, T. Roughgarden, E. Tardos, and V. V. Vazirani, Algorithmic Game Theory. New York, NY, USA: Cambridge University Press, 2007.

[18] A.J.G. Anandkumar, A. Anandkumar, S. Lambotharan and J. Chambers, "Robust rate-maximization game under bounded channel uncertainty," submitted to IEEE Transactions on Signal Processing, eprint at arXiv:1011.1566.

[19] S. Hayashi and Z.-Q. Luo, "Spectrum management for interferencelimited multiuser communication systems," IEEE Transactions on Information Theory, vol. 55, pp. 1153-1175, Mar. 2009. 